\title{
ANÁLISE DA IMPLANTAÇÃO DO PRONTUÁRIO ELETRÔNICO DO CIDADÃO (PEC): REVISÃO DE LITERATURA
}

\author{
ANALYSIS OF THE IMPLEMENTATION OF THE CITIZEN'S \\ ELECTRONIC CHART (CEC): LITERATURE REVISION
}

\author{
Mariana Pinheiro de Sousa ${ }^{1}$ \\ Ana Paula Rocha Sá ${ }^{2}$ \\ Júlia Andrade Farias ${ }^{3}$ \\ Gabrielle Letícia Albuquerque 4 \\ Nicolly Andrade Cavalcanti ${ }^{5}$ \\ Cristiane Falcão de Almeida ${ }^{6}$
}

RESUMO: OBJETIVO: Analisar os avanços e desafios da implantação do Prontuário Eletrônico do Cidadão (PEC) no Sistema Único de Saúde (SUS). MÉTODO: Realizou-se a pesquisa em banco de dados eletrônicos: Google Acadêmico, Microsoft Academic, Biblioteca Virtual em Saúde, Arca, Academos Revista Científica da Saúde, aplicando os descritores: "Prontuário Eletrônico do Cidadão", "Atenção Básica" e "Gestão de Saúde". Foram utilizados artigos escritos em português, no período de 2013 a 2020 com posterior refinamento dos resultados. RESULTADOS: Foram analisados 10 artigos científicos seguindo os critérios de inclusão e exclusão delineados, em que todos buscavam efetivamente estabelecer o objetivo principal da implementação do prontuário eletrônico pelo Ministério da Saúde que é facilitar o manejo das informações dos paciente buscando a melhoria do acesso e do atendimento em saúde. Todavia, dificuldades como a desproporcionalidade de infraestrutura adequada para atendimento do cidadão, bem como resistência por parte de alguns profissionais também foram observadas. CONCLUSÃO: Apesar das dificuldades encontradas, é notório o avanço do sistema. Portanto, acredita-se que através da equipe interligada pelo PEC é possível proporcionar, de forma multidisciplinar, um cuidado integral de melhor qualidade.

\footnotetext{
${ }^{1}$ Acadêmico de Medicina da Unifacisa de Campina Grande.

2 Acadêmico de Medicina da Unifacisa de Campina Grande.

${ }^{3}$ Acadêmico de Medicina da Unifacisa de Campina Grande.

${ }^{4}$ Acadêmico de Medicina da Unifacisa de Campina Grande.

${ }^{5}$ Acadêmico de Medicina da Unifacisa de Campina Grande.

6 Docente do Centro Universitário Unifacisa de Campina Grande. Mestrando do Programa de Pósgraduação em Saúde Coletiva da Universidade Estadual da Paraíba.
} 
Palavras chave: Atenção Básica. Gestão de Saúde. Prontuário Eletrônico do Cidadão.

ABSTRACT: OBJECTIVE: To analyze the advances and challenges of the implementation of the Citizen's Electronic Chart (CEC) in the National Health Service (NHS). METHOD: The research was carried out in the following electronic databases: Google Scholar, Microsoft Academic, Virtual Heath Library, Ark and Academos Scientific Journal of Health, using the descriptors: "Citizen's Electronic Chart", "Primary Care", and "Health Management". Scientific papers written in portuguese from 2013 to 2020 were chosen, with further refinement of the results. RESULTS: 10 scientific papers were analyzed according to the inclusion and exclusion criteria previously outlined. It was noticed that these papers aimed to effectively establish the main objective of the implementation of the Citizen's Electronic Chart by the Ministry of Health, which is to facilitate the management of patient data seeking to improve access and health care. However, difficulties such as the disproportionality of adequate infrastructure to serve the citizen, as well as resistance by some professionals were also observed. CONCLUSION: Despite the difficulties encountered, the system's progress is notorious. Therefore, it is believed that through the interconnected team by the CEC it is possible to provide, in a multidisciplinary way, comprehensive care of better quality.

Keywords: Primary Care. Health Management. Citizen's Electronic Chart. 


\section{INTRODUÇÃO}

Os registros em papel estão sendo substituídos pelos registros eletrônicos em um processo que parece irreversível. Essa alteração na dinâmica de arquivamento de informações acabou por alcançar profissionais e instituições da área de saúde, as quais buscam incorporar essas tecnologias digitais a fim de catalogar as suas atividades cotidianas. Logo, a organização das atividades tem sofrido mudanças significativas e consequentemente a forma como as pessoas e as instituições lidam com essas informações (DIAS et al., 2013).

Nesse contexto encaixa-se o Prontuário Eletrônico do Cidadão (PEC), definido como um sistema de prontuário médico padronizado e digital, onde todas as informações de saúde, sejam elas clínicas ou administrativas, ao longo da vida de um indivíduo estão armazenadas. O acesso rápido aos problemas de saúde e intervenções atuais, a recuperação de informações clínicas e a possibilidade de ser sistema de apoio à decisão médica podem ser citadas como exemplos de suas principais características (BRASIL, 2016).

Em se tratando de sua implantação, o Prontuário Eletrônico do Cidadão foi instituído sob a resolução no 7 de 24 de novembro 2016 pelo Ministério da Saúde, considerando a necessidade de obter informações integradas sobre a atividade assistencial desenvolvida pela Atenção Básica no território nacional, visando subsidiar a gestão, planejamento, investigação clínica e epidemiológica e a avaliação dos serviços de saúde (BRASIL, 2016).

Essa reestruturação dos Sistemas de Informação a Saúde, através do PEC, objetiva atender amplamente às Unidades Básicas de Saúde (UBS) que já possuem estruturas de informatização, seja total ou parcialmente, de forma a integrá-los e a fornecer suporte adequado aos profissionais envolvidos no atendimento do paciente (BRASIL, 2012). Em outras palavras, os diversos sistemas de informação passaram a unificar-se, promovendo a facilitação e aumento da confiabilidade em relação às informações referentes à saúde como um todo. 
Tendo em vista o avanço quanto à praticidade do acesso aos dados da saúde promovido pelo Prontuário Eletrônico do Cidadão, é notória a sua significância no progresso dos Sistemas de Informação e, como conseguinte, na qualificação do cuidado em saúde. Segundo Lima et al. (2018), a utilização de registros eletrônicos acaba por facilitar o acompanhamento dos dados clínicos do usuário na Rede de Atenção em Saúde (RAS), uma vez que estes não devem ser mais fragmentados, da forma como ocorre com os registros em papel, mas devem estar integrados. Assim, o prontuário eletrônico auxilia no atendimento integral do cidadão por promover uma assistência mais eficiente.

Levando-se em consideração a importância do PEC anteriormente explanada e o fato de ainda haver poucos artigos disponíveis na literatura que contemplem seus mais variados aspectos, ratifica-se a realização do presente estudo, o qual objetiva compilar as informações presentes nos bancos de dados eletrônicos utilizados, de forma a analisar a implantação desse sistema de registro, sua abrangência e significância para a melhoria do armazenamento de dados da saúde, além dos avanços e desafios por ele encontrados para que seja amplamente integrado ao Sistema Único de Saúde e validado perante a sociedade.

\section{METODOLOGIA}

Procedeu-se a pesquisa em banco de dados eletrônicos: Google Acadêmico, Microsoft Academic, Biblioteca Virtual em Saúde, Arca, Academos Revista Científica da Saúde, utilizando os descritores: "Prontuário Eletrônico do Cidadão", "Atenção Básica" e "Gestão de Saúde". Foram utilizados artigos escritos em português, no período de 2013 a 2020, pelo fato do e-SUS AB ter sido criado em 2013, procurando-se fazer uma revisão de literatura desde sua implementação até a atualidade.

Após a primeira triagem através da leitura dos títulos foi verificada a existência de duplicidade dos artigos nas seleções nos bancos de dados, ou seja, 
artigos iguais foram selecionados em bancos de dados diferentes, sendo assim excluídos.

Os artigos foram selecionados a partir da leitura dos resumos das publicações a fim de refinar a amostra por meio de critérios de inclusão e exclusão. Foram incluídos artigos científicos, artigos originais, estudos de caso, relatos de experiência e um estudo intervencional, os quais demonstram a análise do uso do PEC nas Unidades de Saúde do Brasil, bem como sua importância e implicações para a saúde, para os usuários e para os profissionais de saúde. Foram excluídos artigos que não tratassem diretamente do Prontuário Eletrônico do Cidadão, ou que apenas expressassem como se dava o uso da ferramenta. Por meio desse processo, a amostra final foi constituída por 10 artigos.

A avaliação crítica dos artigos consistiu na leitura íntegra de todos os artigos selecionados. Foi elaborado um quadro com os principais eixos temáticos por meio da leitura e releitura dos resultados dos estudos, procurando identificar aspectos relevantes que se repetiam ou se destacavam.

\section{RESULTADOS}

Nesta pesquisa foram analisados 10 artigos científicos seguindo os critérios de inclusão e exclusão delineados anteriormente. Para facilitar a análise e apresentação dos resultados foi feito o Quadro 1, com informações acerca do tipo de estudo e de seu eixo temático principal de cada estudo. 
Quadro 1 - Tipo e eixo temático principal de cada estudo.

\begin{tabular}{|c|c|c|}
\hline TÍTULO & DELINEAMENTO & EIXO TEMÁTICO \\
\hline $\begin{array}{l}\text { Uso do prontuário eletrônico do } \\
\text { cidadão na atenção primária: } \\
\text { relato de experiência }\end{array}$ & o & $\begin{array}{l}\text { ussões da implantação do } \\
\text { rio eletrônico do cidadão nas } \\
\text { es Básicas de Saúde }\end{array}$ \\
\hline $\begin{array}{|lcc|}\text { Avaliação } & \text { das } & \text { capacitações } \\
\text { realizadas pelo TelessaúdeRS } \\
\text { para a utilização do Prontuário } \\
\text { Eletrônico do Cidadão (PEC) }\end{array}$ & $A$ & $\begin{array}{l}\text { Análise de capacitações profissionais } \\
\text { para aprender a usar o PEC }\end{array}$ \\
\hline \begin{tabular}{|lrr|} 
Avaliação de & \multicolumn{2}{c}{ produção } \\
audiovisual como objeto de \\
aprendizagem & virtual paralA \\
utilização do Prontuário Eletrônico \\
do Cidadão (PEC)
\end{tabular} & & fissionais \\
\hline $\begin{array}{l}\text { Prontuário eletrônico do cidadão: } \\
\text { Desafios e superações no } \\
\text { processo de informatização }\end{array}$ & & $\begin{array}{l}\text { Repercussões da implantação do } \\
\text { prontuário eletrônico do cidadão nas } \\
\text { Unidades Básicas de Saúde }\end{array}$ \\
\hline $\begin{array}{|lcc|}\text { Valorização do } & \text { adequado } \\
\text { preenchimento do prontuário E } & \text { do } \\
\text { eletrônico do cidadão na ESFir } \\
\text { Ernesto Araújo }\end{array}$ & $\begin{array}{l}\text { clín } \\
\text { encional }\end{array}$ & \begin{tabular}{|lcll} 
Importância & do & PEC & na \\
documentação & dos & dados & do \\
paciente & & &
\end{tabular} \\
\hline $\mid \begin{array}{lll}\text { A importância do prontuário } \\
\text { eletrônico } \\
\text { municípios }\end{array}$ & áfica & $\begin{array}{l}\text { Ressalta a importância e a eficiência } \\
\text { do uso do prontuário eletrônico, bem } \\
\text { como suas vantagens } \\
\text { desvantagens. }\end{array}$ \\
\hline $\begin{array}{l}\text { Relato da implantação do Sistema } \\
P E C \text { e-SUS AB nas unidades de } \\
\text { atenção primária da AP 1.0, no } \\
\text { Município do Rio de Janeiro }\end{array}$ & & $\begin{array}{l}\text { experiência da implantação } \\
\text { tuário eletrônico em unidades } \\
\text { le Janeiro. }\end{array}$ \\
\hline \begin{tabular}{|l} 
A transição do prontuário do \\
paciente em suporte papel para o \\
prontuário eletrônico do paciente \\
e seu impacto para os \\
profissionais de um arquivo de \\
instituição de saúde
\end{tabular} & & $\begin{array}{l}\text { Avaliar o impacto da tecnologia, para } \\
\text { os profissionais de saúde, em } \\
\text { unidades de saúde que antes } \\
\text { possuíam prontuários manuais }\end{array}$ \\
\hline $\begin{array}{l}\text { Prontuário eletrônico - uma } \\
\text { estratégia de coordenação entre a } \\
\text { atenção primária e secundária à } \\
\text { saúde no município de Belo } \\
\text { Horizonte }\end{array}$ & & $\begin{array}{l}\text { Avaliar a contribuição do prontuário } \\
\text { para melhora da comunicação e } \\
\text { continuidade de atendimento da } \\
\text { atenção primária ao serviço } \\
\text { especializado. }\end{array}$ \\
\hline \begin{tabular}{|l|l|} 
Prontuário eletrônico do cidadão e \\
gerência do cuidado de \\
enfermagem na Atenção Básica: \\
um estudo de caso
\end{tabular} & & $\begin{array}{l}\text { Repercussões da implantação do } \\
\text { prontuário eletrônico do cidadão nas } \\
\text { Unidades Básicas de Saúde }\end{array}$ \\
\hline
\end{tabular}

Fonte: Elaborado pelos autores. 
Quanto aos aspectos metodológicos, dentre os 10 artigos selecionados, 4 são estudos de casos, 2 são artigos científicos, 1 é revisão bibliográfica, 1 é 0 artigo original, 1 é um estudo clínico intervencional e 1 é um relato de experiência.

Levando em consideração toda a abrangência dos artigos selecionados bem como o delineamento utilizado para a realização dos estudos, todos buscam efetivamente estabelecer o objetivo principal da implementação do prontuário eletrônico pelo Ministério da Saúde que é facilitar o manejo das informações dos paciente buscando a melhoria do acesso e do atendimento em saúde.

Como demonstrado em vários desses, a digitalização dos dados, levando em consideração dados pessoais, antecedentes de atendimentos, condutas médicas, exames já realizados, medicamentos em uso vigente, dentre várias outras informações, otimizam e aceleram o atendimento dos usuários e a resolução de suas patologias. Além de diminuir a sobrecarga dos espaços que antes eram necessários para armazenar os papéis e pastas dos prontuários manuais, e diminuir também a sobrecarga dos profissionais que precisavam escrever as informações manualmente, e depois localizar esses quando fossem requisitados para eventuais atendimentos e retornos médicos.

\section{DISCUSSÃO}

No Brasil, o Ministério da Saúde por meio do Departamento de Atenção Básica, vem reunindo esforços para reestruturar as informações da atenção primária em saúde modernizando sua plataforma tecnológica, com o objetivo de informatizar as unidades básicas de saúde. Assim, o PEC veio com o intuito de ampliar o cuidado e melhorar o acompanhamento da gestão (BRASIL, 2012).

Em 2018, 43 milhões de brasileiros estavam com seus dados registrados digitalmente por meio de Prontuário Eletrônico do Cidadão. Porém, no mesmo ano, ainda 59\% das Unidades Básicas de Saúde não utilizavam o PEC, o equivalente a 24,4 mil serviços sem cobertura (BRASIL, 2018). 
Apesar de haver um extenso caminho pela frente, houve avanço nos números já que até 2017,64\% das Unidades Básicas de Saúde não utilizavam prontuário eletrônico, totalizando 27,3 mil serviços. No país, só 30 milhões de brasileiros tinham seus dados registrados digitalmente (CONASS, 2017).

Nessa perspectiva, o PEC não pode ser visualizado como um produto, mas sim como um processo no qual novas funções ou recursos podem ser adicionados para melhorar sua capacidade de apoio (MARIN, 2018), assim, de acordo com Veloso et al. (2018), observou-se com a ferramenta uma capacidade de acesso rápido às informações e intervenções realizadas, melhoria na efetividade do cuidado e na qualidade das informações.

\section{Avanços}

Cardoso et al. (2017) relatam que a implantação do PEC traz uma melhor qualidade na assistência à saúde por meio de seus recursos como acesso remoto e simultâneo da informação, melhor legibilidade, integração com outros sistemas de informação, assistência à pesquisa, facilidade no resgate de dados para análise e melhor gerenciamento do cuidado.

Ainda é válido ressaltar a maneira como garante uma documentação segura, sem riscos de perda, totalmente legível, de fácil acesso a qualquer profissional de saúde da equipe, com maior capacidade de armazenamento (HORN, 2017).

Com última atualização do e-SUS $A B$, em 2018, os usuários cadastrados passaram a receber notificações de consultas agendadas. Ademais, os profissionais de saúde também poderão registrar no prontuário eletrônico as vacinas aplicadas, permitindo armazenar todas as ações de saúde do cidadão em um mesmo local. A medida possibilita acessar o histórico do usuário em todos os serviços do SUS que estejam informatizados (BRASIL, 2018).

Outro ponto importante verificado na literatura é a realização de capacitações, extremamente necessárias para a devida instrução, realizadas de diferentes formas. Foram observadas por Pinheiro et al. (2015) formas complementares de educação, 
como a produção de Video Interactive Learning Objects (VILOs), que ensinam o uso do prontuário. Os VILOs são vídeos interativos, de cinco a dez minutos, que abordam objetos de aprendizagem específicos. Essas atividades mostraram-se bastante satisfatórias pelas equipes capacitadas.

\section{Deficiências}

Em contraponto aos avanços, um dos pontos de deficiência observados na literatura foi a consulta na atenção básica, a qual passou a exigir maior tempo (Veloso et al., 2018). Todavia, segundo Gambi et al. (2013), o contrário foi observado, e os profissionais relataram uma consulta muito mais ágil. Essa discrepância relaciona-se ao nível de capacitância do condutor, sofrendo mais delongas os atendimentos com condutores menos experientes.

Levando em consideração a extensão territorial do Brasil e as distâncias geográficas de cada região, existe a dificuldade e desproporcionalidade de infraestrutura adequada para atendimento do cidadão (DIAS et al., 2018).

De acordo com o feedback solicitado pelo Ministério da Saúde, em 2016, as justificativas registradas pelos municípios para não implantação do prontuário eletrônico foram: 84,9\% (21.205) - insuficiência de equipamentos; 73,9\% (18.493) conectividade; 75\% (18.750) - baixa qualificação no uso do PEC; e 67,9\% (16.989) falta de apoio de Tecnologia da Informática (CONASS, 2016).

O alto custo para implementação e manutenção da ferramenta em sistemas nacionais de saúde é um realidade (DIAS et al., 2013). O investimento para implantação do PEC em cada UBS, conforme Manual de implantação do e-SUS AB, é de aproximadamente $R \$ 50.000,00$, só de equipamentos e estruturação de rede lógica para conectividade, variando de acordo com a estrutura de cada UBS (BRASIL, 2014). Entretanto, a gestão governamental trabalha com uma possibilidade de economia em torno de $\mathrm{R} \$ 22$ bilhões por ano para o SUS (BRASIL, 2018).

Ademais, outra desvantagem presente foi uma resistência da equipe de profissionais. Nessa perspectiva, Bezerra (2009) e Massad et al. (2003) apontam a 
possibilidade de uma resistência em alterar rotinas de trabalho quando da passagem dos registros para o meio eletrônico com o esclarecimento de que os registros de papel são familiares aos usuários que, consequentemente, não precisam adquirir novas competências ou comportamentos para usá-los (GAMBI et al., 2013).

Considera-se importante, então, que gestores criem espaços de discussão com as equipes, com o objetivo de identificar necessidades de desenvolvimento e possíveis melhorias no sistema, no processo de trabalho e no próprio registro (GAMBI et al., 2013). O governo e as UBS podem, também, manter uma relação mútua dando seguimento com o feedback por meio das justificativas das unidades possibilitando elencar essas necessidades em prioridades, e assim constituir um plano de ação para resolvê-las.

\section{CONCLUSÃO}

É notório as dificuldades enfrentadas por esse processo de informatização, visto que existe falta de equipamentos, de técnicos de informática para manutenção destes e, principalmente, a necessidade de qualidade de uma rede de internet adequada para proporcionar a interoperabilidade do prontuário. Há também resistência por parte de profissionais ao sistema, uma vez que alguns têm dificuldade de manusear os equipamentos e programas.

No entanto, essa evolução do sistema é bastante relevante, visto que representa um avanço significativo nos processos de trabalho, o qual possibilita melhor organização, sistematização e comunicação das informações de cuidado do paciente, possibilidade de agendamentos, relatórios para análise e desenvolvimento de atividades e integralidade do atendimento, pois acredita-se que articula a comunicação entre os profissionais da atenção primária com os profissionais dos outros níveis de atenção. Portanto, através da equipe interligada pelo PEC é possível proporcionar, de forma multidisciplinar, um cuidado integral de melhor qualidade. 


\section{REFERÊNCIAS BIBLIOGRÁFICAS}

BRASIL. Comissão Intergestores Tripartite. Resolução n. 7, de 24 de novembro de 2016. Define o prontuário eletrônico como modelo de informação para registro das ações de saúde na atenção básica e dá outras providências. Brasília: Comissão Intergestores Tripartite, 2016. Disponível em: http://bvsms.saude.gov.br/bvs/saudelegis/cit/2016/res0007_24_11_2016.html. Acesso em: 29 fev. 2020.

DIAS, Maura Pereira; GIOVANELLA, Ligia. Prontuário eletrônico - uma estratégia de coordenação entre a atenção primária e secundária à saúde no município de Belo Horizonte. Reciis, Rio de Janeiro, v. 7, n. 2, p.1-17, ago. 2013.

ESTEVAM DIAS, Monica Aguilar et al. Relato da implantação do Sistema PEC e-SUS AB nas unidades de atenção primária da AP 1.0, no Município do Rio de Janeiro. Academus Revista Científica da Saúde, [S.I.], v. 3, n. 2, p. 36-43, set. 2018.

FERREIRA, Janise Braga Barros. A transição do prontuário do paciente em suporte papel para o prontuário eletrônico do paciente e seu impacto para os profissionais de um arquivo de instituição de saúde. Reciis, Rio de Janeiro, v. 7, n. 7, p.1-15, jun. 2013.

HORN, Roberta de Freitas. Valorização do adequado preenchimento do prontuário eletrônico do cidadão na ESF Ernesto Araújo. 2017. 18 f. TCC (Graduação) - Curso de Curso de Especialização em Saúde da Família, Unasus/ufscpa, Porto Alegre, 2017.

IVY TECH COMMUNITY COLLEGE. Video Interactive Learning Objects. Ivy Tech [Internet]. 2013. Disponível em: http://www.sdccdonline.net/faculty/otes/VilosBrochure.pdf . Acesso em: 23 fev. 2020.

LIMA, Verineida Sousa et al. PRONTUÁRIO ELETRÔNICO DO CIDADÃO: DESAFIOS E SUPERAÇÕES NO PROCESSO DE INFORMATIZAÇÃO. Anais do V Sinforgeds, Fortaleza, p.216-231, jun. 2018.

OLIVEIRA, Priscila Sanches Aquino de. Prontuário Eletrônico do Cidadão e gerência do cuidado de enfermagem na atenção básica: um estudo de caso. 2019. $146 \mathrm{f}$. Tese (Doutorado) - Curso de Enfermagem, Universidade Federal de Juiz de Fora, Juiz de Fora, 2019.

PINHEIRO, João Paulo et al. Avaliação das capacitações realizadas pelo TelessaúdeRS para a utilização do Prontuário Eletrônico do Cidadão (PEC). Anais - Qualihosp 2015, São Paulo, p.606-607, abr. 2015.

PINHEIRO, João Paulo et al. Avaliação de produção audiovisual como objeto de aprendizagem virtual para utilização do Prontuário Eletrônico do Cidadão (PEC). Anais - Qualihosp 2015, São Paulo, p.602-603, abr. 2015.

VELOSO, Lorena Uchôa Portela et al. USO DO PRONTUÁRIO ELETRÔNICO DO CIDADÃO NA ATENÇÃO PRIMÁRIA: relato de experiência. Anais do I Congresso Norte Nordeste de Tecnologias em Saúde, Teresina, v. 1, n. 1, dez. 2018. 